\title{
Interactive comment on "Effects of interannual variability in snow accumulation on energy partitioning and surface energy exchange in a high-Arctic tundra ecosystem" by C. Stiegler et al.
}

Anonymous Referee \#2

Received and published: 25 April 2016

In this study the authors assess the impact of strong inter-annual variability in snow accumulation during two subsequent years $(2013,2014)$ on the land-atmosphere interactions and surface energy exchange in two well instrumented high-Arctic tundra ecosystems under different moisture regimes (wet fen and dry heath) in Zackenberg, Northeast Greenland. The study takes advantage of the natural laboratory conditions of strongly different snowcover regimes between the two years, which motivates this study.

Their results suggest that in a changing climate with higher temperature and more precipitation the surface energy balance of this high-Arctic tundra ecosystem may experience a further increase in the inter-annual variability of energy accumulation, par- 
titioning and redistribution.

I think the experimental setup is nice and clear with two differing ground moisture regimes being complemented by two strongly different snowcover regimes. In addition the paper is well organised and clear with precise method descriptions and a clear analysis. The paper is quite straightforward, primarily describing and interpreting the meteorological measurements in the context of their experimental setup and through seasonal changes (polar night, snowmelt, growing season). Due to the paucity of such measurements in the high Arctic this study is an important contribution to knowledge about atmosphere-surface dynamics in the high Arctic and recommend publishing subject to a few minor comments below.

\section{COMMENTS}

1. p.2 I.28- You need to mention the two site setup early in this paragraph as you just drop 'at our two high-Arctic sites' in at the end rather unexpectedly.

2. P.4 I.6 : this snow depth measurement comes from the Asiaq station or is made directly at the tower? If at the tower what's the instrument? If at the Asiaq station can you comment on representativeness?

3. p.4 I.14 can you mention the soil depths you measured at?

4. I wasn't able to identify which model OTT pluvio you used based on the reference (p.4 I.17). 5. How do you power your stations? Particularly during the polar night?

6. Perhaps a parameter table would be a useful look up for this paper with categories of: units, measured/derived, location, instrument (if measured), temporal resolution etc.

7. I found the last paragraph of Methods (p.6 I.1-10) where you define the "seasons": polar night, melt and growing a little disconnected. Obviously, you organise your results according to these categories which I think is nice, but you could add to this description that this is how you will present the data and why this is informative. This would make 
the 'story' flow a little better.

8. I feel like the conclusions are missing some kind of outlook to what next ie. integrating models to scale results/ investigate other aspects of the enrgy balance or strategies to reduce the energy balance closure problem. I think a few sentences reflecting on ways of building on this study with further work would be useful.

9. Figure 1a: can you mark the Asiaq station on the map?

10. Figure 2: mention which site this is in the caption.

11. Picking up on the comments of $\mathrm{J}$. McFadden and while I agree the stacked plots (Figure 3a/b) make it tricky to identify trends in individual years - I think the key point the authors intend to show is the cumulative energy inputs from all components. If that's the intention I would say some form of cumulative presentation is important.

12. Not immediately obvious which lines the axis refer to in Figure $6 \mathrm{~b}$ - soil moisture is indicated on the black line, perhaps can do the same with blue/red lines (evaporation?).

Interactive comment on The Cryosphere Discuss., doi:10.5194/tc-2016-5, 2016. 